10-ORIGINAL ARTICLE

CLINICAL INVESTIGATION

\title{
Structural changes of dilated pelvic ureters in adults ${ }^{1}$
}

\author{
Fernando Nevesi, Waldemar Silva Costa ${ }^{\mathrm{II}}$, Jose Genilson Ribeiro" ${ }^{\mathrm{III}}$, Marcio Antonio Babinski ${ }^{\mathrm{IV}}$, Carla Braga Mano Gallov, \\ Francisco José Barcellos SampaiovI
}

DOI: http://dx.doi.org/10.1590/S0102-86502014001700010

${ }^{\mathrm{I}} \mathrm{PhD}$, Urogenital Research Unit, State University of Rio de Janeiro (UERJ), Rio de Janeiro-RJ, Brazil. Performed the experiment, acquisition and interpretation of data, wrote the manuscript.

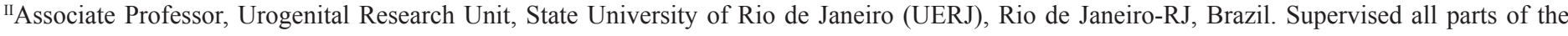
experiment, statistical analysis, read and approved the final manuscript.

IIIPhD, Fluminense Federal University (UFF), Niteroi-RJ, Brazil. Acquisition of data, manuscript preparation.

${ }^{\text {IV }} \mathrm{PhD}$, Fluminense Federal University (UFF), Niteroi-RJ, Brazil. Acquisition and interpretation of data, manuscript preparation.

${ }^{\mathrm{P}} \mathrm{PhD}$, Urogenital Research Unit, State University of Rio de Janeiro (UERJ), Rio de Janeiro-RJ, Brazil. Acquisition of data, manuscript preparation.

${ }^{\mathrm{VI} F u l l}$ Professor, CNPq 1A researcher, Urogenital Research Unit, State University of Rio de Janeiro (UERJ), Rio de Janeiro-RJ, Brazil. Designed the study, critical revisions, read and approved the final manuscript.

\section{ABSTRACT}

PURPOSE: To perform a quantitative assessment of different types of pelvic ureter tissues with chronic dilation in adults, using stereological methods.

METHODS: We analyzed fragments of dilated pelvic ureters obtained from 6 patients aged between 35 and 67 years (mean, 46 years) who underwent ureteral reimplantation surgery for different reasons. The obstruction duration ranged from 27 to 180 days (mean, 93 days). The control group included fragments of normal pelvic ureters obtained during nephrectomy in 7 kidney transplant donors (age: range, 25-51 years; mean, 36 years). The volumetric density of collagen in the ureter, elastic fibers, and smooth muscle fibers was assessed.

RESULTS: The volumetric density (Vv) of collagen showed no significant difference between the two groups (control: $45.3 \pm 6.1$; dilated: $40.8 \pm 6.9 ; \mathrm{P}=0.23$ ). A statistically significant increase in $\mathrm{Vv}$ of elastic fibers was observed in the dilated ureters (control: 18.4 \pm 1.2 ; dilated: $24.6 \pm 5.4 ; \mathrm{P}=0.03$ ). A statistically significant increase in the $\mathrm{Vv}$ of smooth muscle fibers was observed in the dilated ureter (control: $42.0 \pm 6.0$; dilated: $56.2 \pm 6.1 ; \mathrm{P}=0.001$ ).

CONCLUSION: The chronically dilated pelvic ureters had significantly more elastin and smooth muscle contents than the controls.

Key words: Ureter. Stereology. Elastic fibers. Connective tissue. Smooth muscle cells. 


\section{Introduction}

The ureter diameter narrows along its length; including the ureteropelvic junction, iliac vessel crossing, and ureterovesical junction, with the latter being the most narrow ${ }^{1}$. This physiological narrowing of the ureter forms zones, with high chances of obstruction or interruption of urine flow, often resulting in the dilatation of regions of the organ proximal to the obstruction ${ }^{1,2}$.

Many illnesses may compromise the normal functioning of the ureter, resulting in an increase in intraluminal pressure and a decrease in glomerular filtration ${ }^{2}$. The chronic obstruction of the ureter may cause partial or total loss of kidney function. Although this obstruction can spontaneously resolve or be resolved through treatment, this does not always mean complete functional recovery of the ureter and/or corresponding renal unit ${ }^{3}$.

Among studies on ureteral morphology, only that of Stein and Weinberg ${ }^{4}$ showed quantitative data for adults. Most studies compared normal and dilated ureters in infants with congenital disorders ${ }^{5-13}$. None of these studies assessed adults with dilated ureters resulting from acquired illnesses.

The aim of this study was to performa quantitative assessment, using stereological methods, of different tissues of the dilated pelvic ureter in adults. Determining these elements may contribute to the better understanding of these mechanisms that regulate the transport of urine from the kidney to the bladder, as well as assist the surgeon in the removal of obstructions or correction of lesions.

\section{Methods}

Fragments of dilated pelvic ureters was obtained from 6 patients ( 5 men and 1 woman) aged between 35 and 67 years (mean age, 46 years) who underwent ureter reimplantation surgery for different reasons as follows: distal ureteral calculi $(n=2)$, postureterolithotomy stenosis $(n=1)$, bladder neoplasia $(n=2)$, and pelvic endometriosis $(n=1)$. The obstruction duration ranged from 27 to 180 days (mean, 93 days). Using samples obtained from patients with obstruction due to bladder neoplasia, freeze biopsy confirmed the absence of neoplasia in the ureteral stump.

The control group consisted of fragment samples of normal pelvic ureters obtained during nephrectomy in 7 male kidney transplant donors aged between 25 and 51 years (mean, 36 years). In both groups, the segment analyzed measured approximately $5 \mathrm{~mm}$ in length.

This study was approved by the ethics committee at our institution - State University of Rio de Janeiro - and all the patients signed a declaration of informed consent.
The tissue samples obtained were fixed in buffered formalin ( $\mathrm{pH}$ 7.2) and processed according to the paraffin inclusion protocol for formalin-fixed paraffin-embedded tissue samples. The prepared specimens were then divided into $5-\mu \mathrm{m}$ thick tissue sections and stained with hematoxylin and eosin for analysis of their integrity, excluding samples with artefacts. The Picrosirius staining technique was used, and the sections were examined under a polarized light microscope to characterize different types of collagen. The van Gieson staining technique was used to quantify the total collagen and smooth muscle contents, as these two components show significant differences in staining with this technique.

For the immunohistochemical analysis, the avidinbiotin method was used to identify elastic fibers using primary anti-elastin antibodies (Sigma Immunochemicals, St. Louis, USA). All the primary antibodies were used simultaneously. Tissue fragments for which the primary antibody was substituted with phosphate-buffered saline were used as negative controls, and those with specific antigens were used as positive controls. The immunostaining was revealed in a solution of $3,3^{\prime}$-dichlorobenzidine tetrahydrochloride in $0.1 \% \mathrm{H}_{2} \mathrm{O}_{2}$, washed in distilled water, dehydrated in increasing ethanol concentrations, diaphonized in xylol, and mounted with ethelan.

For the morphometric and stereological analyses, 10 fragments of 5 different sections with a gap of $50 \mu \mathrm{m}$ were selected for each sample. Four sections were analyzed for each fragment, for a total of 200 sections. The sections were analyzed under an Olympus light microscope coupled to a Sony chargecoupled device digital camera, with the microscopic images being transmitted to a Sony KX14-CP1 monitor.

The stereological analysis was performed using a M42 test system ${ }^{14}$. The M42 system is composed of 21 line segments with 42 test points. The stereological parameter analyzed was volumetric density $(\mathrm{Vv}): \mathrm{Vv}=\mathrm{Pp} / \mathrm{Pt} \%$, with $\mathrm{Pp}$ being the points relating to the smooth muscle, collagen, or elastic fibers, and $\mathrm{Pt}$ being the total test points of the test system ${ }^{15,16}$.

The difference between the groups was analyzed using the software Graph Prism 3.0. The nonparametric Mann-Whitney $\mathrm{U}$ test was used to compare the groups. The numerical results were presented as mean \pm standard deviation values, and the difference was considered as statistically significant when $\mathrm{p}<0.05$.

\section{Results}

The tissue sections of the materials stained with Picrosirius and observed in a microscope under polarized light 
showed that both normal and dilated ureters have different types of collagen, characterized by different colors from red to orange to green (Figures $1 \mathrm{~A}$ and $1 \mathrm{~B}$ ).
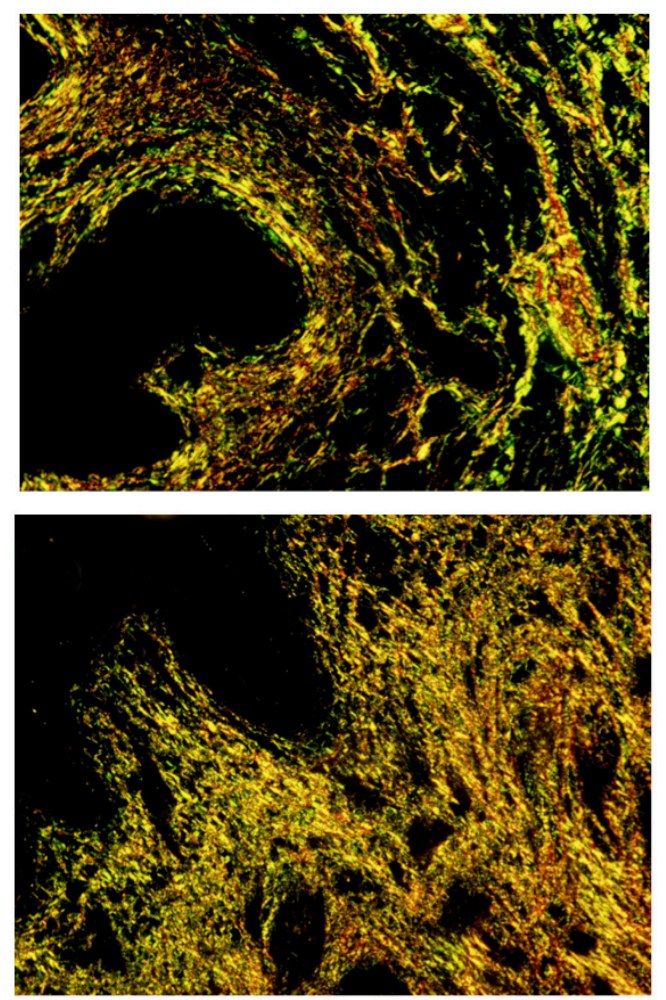

Figure 1 - A) Control ureter. The yellow-red stain predominates over the other stain colors. B) Dilated ureter. A predominant green color could be observed. Picrosirius staining (original magnification $\times 200$ ).

No significant difference in volumetric density ( $\mathrm{Vv}$ ) of collagen was observed between the two groups, analyzed with sections stained using the van Gieson technique (Table 1, and Figures $2 \mathrm{~A}$ and $2 \mathrm{~B}$ ). With regard to the tissue sections stained using the van Gieson technique, a significant increase in volumetric density of smooth muscle fibers was observed between the dilated ureters and the controls (Table 1, and Figures 2A and 2B).

A significant increase in elastic system fibers was observed in the dilated ureters by using immunostaining technique with anti-elastin antibody (Table 1, and Figures 3A and 3B).

Table 1 - Results of the quantitative analysis (volumetric density $[\mathrm{Vv}]$ ) of the histological elements of the ureters in the control and dilated ureter groups

\begin{tabular}{|c|c|c|c|}
\hline Structure & Control Vv\% & Dilated Vv\% & $\mathbf{P}$ \\
\hline Collagen & $45.3 \pm 6.1$ & $40.8 \pm 6.9$ & $0.23 *$ \\
\hline Elastic fibers & $18.4 \pm 1.2$ & $24.6 \pm 5.4$ & $0.03^{\dagger}$ \\
\hline Smooth muscle fibers & $42.0 \pm 6.0$ & $56.2 \pm 6.1$ & $0.001^{\dagger}$ \\
\hline \multicolumn{4}{|c|}{$\begin{array}{l}\text { The data are represented as mean } \pm \text { standard deviation values. } \\
* \text { Not statistically significant by Mann-Whitney } U \text { test. } \\
+ \text { Statistically significant by Mann-Whitney } U \text { test. }\end{array}$} \\
\hline
\end{tabular}
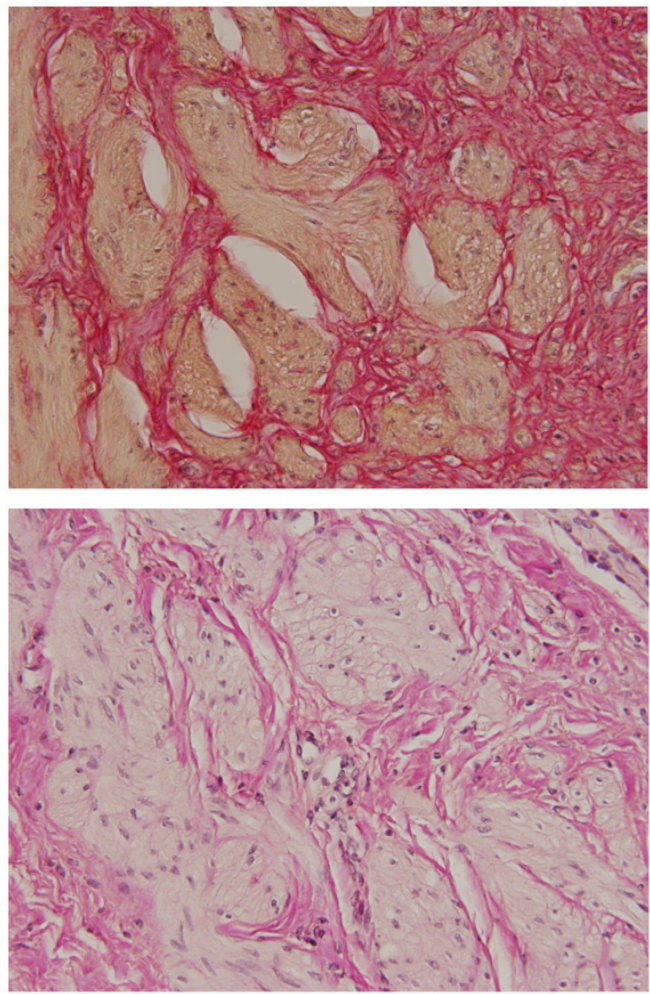

Figure 2 - A) Control ureter. B) A dilated ureter. Connective tissue stained red, and smooth muscle stained yellow. Note the increase in smooth muscle content in the dilated ureter (B), as confirmed from the quantitative analysis. Van Gieson staining (original magnification $\times 400$ ).

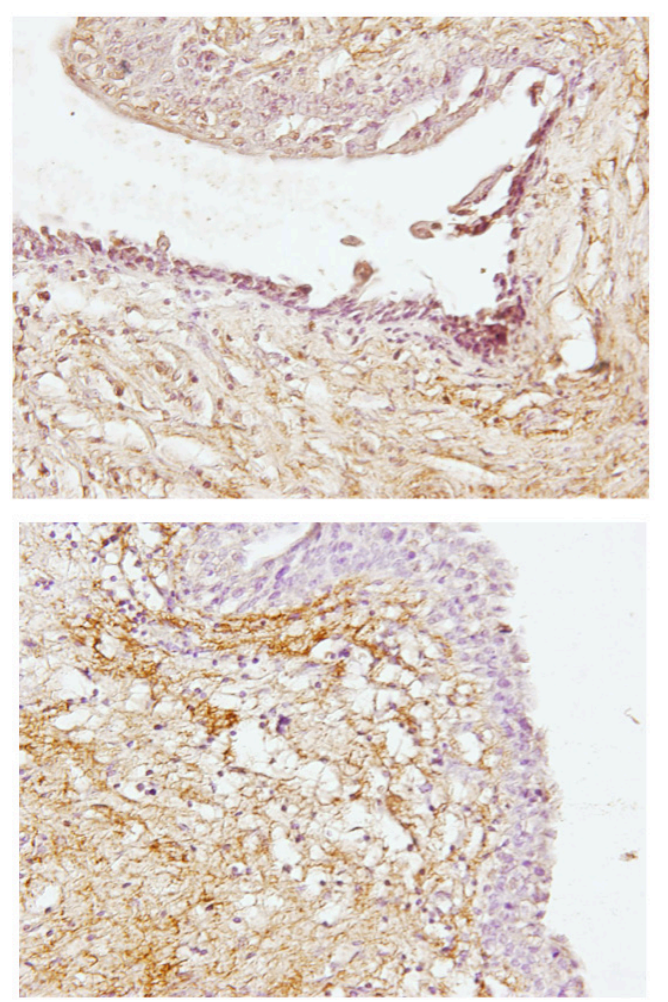

Figure 3 - A) Control ureter. B) A dilated ureter. Connective tissue stained red, and smooth muscle stained yellow. Note the increase in smooth muscle content in the dilated ureter $(\mathbf{B})$, as confirmed from the quantitative analysis. Van Gieson staining (original magnification $\times 400$ ). 


\section{Discussion}

Few studies have characterized the microscopic changes in dilated ureters in adults, as most studies used children or subjective quantitative methods. Therefore, this study aimed to provide data that quantitatively and objectively characterize fibrous elements and smooth muscle fibers of dilated ureters using stereological techniques. This method was preferred because we chose to work with volumetric density (Vv) instead of area density obtained using computational methods. Stereological and computational methods provide objective results and have been used in different studies by our group. In most cases, the computational methods involve the observer choosing a color to be quantified. In the stereological method, the observer counts the number of points of a stereological grid that are superimposed on a specific structure. It is essential that all fields are randomly analyzed and the sample is homogeneous. Both of these conditions were taken into account in our study. The computational methods provide area density and we opted for using volumetric density.

\section{Collagen}

The quantification of collagen in this study was performed using stereological methods, with minimal bias, and showed no statistically significant difference between the control and dilated groups. Stein and Weinberg (4) found a small increase in the amount of connective tissue in dilated ureters. However, in their study, collagen was quantified using a graduation system, from 1 to 4, a frequently used methodology, which has a subjective aspect and may make the interpretation and comparison of results difficult. Therefore, it is possible that the small increase found by these authors, may not be statistically significant.

Generally, in studies where an increase in collagen content was observed, quantitative analyses were performed with ureter samples from populations different from those in this study ${ }^{4,8-10}$. On transmission electron microscopy, Medel and Quesada $^{11}$ analyzed collagen content in normal ureters and in congenitally dilated ureters. A muscle-to-collagen ratio of 2:1 was observed in the normal ureters, and an increase in muscleto-collagen ratio of 3-4:1 was observed in primary obstructive megaureters. The increase in volumetric density of smooth muscle in this study seems to be consistent with the increase in muscle-tocollagen ratio in the study by Medel and Quesada ${ }^{11}$, despite their study population being predominantly children, whereas our study population consisted of adults.
Lee et al. ${ }^{12}$ used histochemical and immunohistochemical techniques to analyze the relative quantities of collagen types I and III. No significant difference in the quantity of any collagen subtype was observed between the primary megaureters and the controls. In the ureters with reflux, however, a decrease in type I collagen content and an increase in type III collagen content were observed. In our collagen analysis using Picrosirius staining under polarized light, significant differences in the distribution and arrangement of collagen fibers were observed between the study groups; however, definitively characterizing the collagen types in each group was not possible.

\section{Elastic fibers}

The immunohistochemical analysis of elastic fibers revealed a statistically significant increase in elastin in the dilated ureters.

In a study on children, Pagano et al. ${ }^{7}$ found an increase in elastin in dilated ureters, which is consistent with our results in adults. However, the authors did not explicitly describe the etiology or obstruction duration. Kim et al. ${ }^{17}$ analyzed the elastin content in proximal ureters in children who underwent pyeloplasty and found a poor postoperative prognosis in patients whose ureters had high quantities of elastin. By analogy, the higher quantity of elastin in dilated pelvic ureters may be associated with a poor postoperative prognosis in patients who had undergone ureter reimplantation surgery.

\section{Smooth muscle fibers}

The study by Stein and Weinberg ${ }^{4}$ was one of the first to provide quantitative data, showing an increase in smooth muscle fibers in dilated ureters. In our study, the analysis of the muscular component also revealed a significant increase in smooth muscle in dilated ureters. Although our findings are consistent with those of Stein and Weinberg ${ }^{4}$, with regard to the increase in smooth muscle content, the percentage increase found in our study is higher than that in their study. This difference is probably due to the chronic nature of the obstruction, which was the case in the patients of our study.

Hanna et al. $^{6}$, who analyzed primary obstructive megaureters, found muscular hypertrophy in both dilated segments and ureters with stenosed segments with less evident alterations than in more clearly altered ureters in their stenosed areas. The same authors ${ }^{8}$ observed muscular hypertrophy when analyzing dilated ureters caused by vesicoureteral reflux and valves of the posterior urethra. 
In a study that analyzed congenital megaureters in adults and children by transmission electron microscopy, it was observed muscular hypertrophy of the dilated part of the ureter ${ }^{11}$. Although these authors, as well as Hanna et al. ${ }^{8}$, did not perform quantitative analyses, the muscular hypertrophy found was, in some ways, consistent with the results of our study. They also verify that long-term ureter obstruction may result in hypertrophy of the muscular layer of the organ.

Gearhart et al. ${ }^{13}$ showed that $61 \%$ of the normal ureter in children is smooth muscle fibers. Our results indicated a proportion of $42.02 \%$ in normal adult ureters. This difference in muscle volumetric density may be due to a decrease or atrophy of musculature with age, which would explain the difference found between children and adults. In our study, the increase in volumetric density $(\mathrm{Vv})$ of the smooth muscle may be due to hypertrophy of the muscle cells and some studies agree with this ${ }^{8,11}$. However, these studies did not determine when or how fast hypertrophy of the musculature occurs and if it returns to initial values once the obstruction is removed. No data have been reported that allow us to say whether the etiology, obstruction duration, or past or present infections influence alterations in the ureter musculature. Therefore, a precise quantitative assessment is not only justifiable but also necessary for the accurate assessment of the consequences of ureteral obstruction. Our hypothesis is that hypertrophy of the musculature occurs to generate greater intraluminal pressures so that urine can flow from the ureter and pass through the obstruction, similar to what happens in the bladder of patients with infravesical obstruction ${ }^{18,19}$. Evidence suggests that the decreased muscle content of dilated ureters is associated with low success rates of ureteral reimplantation surgeries ${ }^{9}$.

We admit that our sample size was small. However, this study involved human materials, which are always difficult to obtain and process.

\section{Conclusion}

The data provided in this study will help to contribute for a better understanding of the mechanisms involved in ureteral dilatation. From our results, we can objectively conclude that the volumetric density of collagen remained unaltered in the dilated ureters, although the elastic and smooth muscle fiber contents were significantly increased.

\section{References}

1. Kabalin JN. Surgical anatomy of the retroperitoneum, kidneys and ureter In: Walsh PC, et al. (eds.), Campbell's Urology. 8th ed. Philadelphia, WB Saunders. 2002; pp. 3-40.
2. Weiss RM. Physiology and pharmacology of the renal pelvis and ureter In: Walsh PC, et al. (ed.), Campbell's Urology. 8th ed. Philadelphia, WB Saunders. 2002; pp. 377-403.

3. Park JM, Bloom DA. The pathophysiology of UPJ obstruction. Current concepts. Urol Clin North Am. 1998 May;25(2):161-9.

4. Stein J, Weinberg SR. A histologic study of the normal and dilated ureter. J Urol. 1962 Jan;87:33-8.

5. Hanna MK, Jeffs RD, Sturgess JM, Barkin M. Ureteral structure and ultrastructure. Part I. The normal human ureter. J Urol. 1976 Dec;116(6):718-24

6. Hanna MK, Jeffs RD, Sturgess JM, Barkin M. Ureteral structure and ultrastructure. Part II. Congenital ureteropelvic junction obstruction and primary obstructive megaureter. J Urol. 1976 Dec;116(6):725-30.

7. Pagano F, Passerini G, Cortivo R, Daga-Gordini D, Abatangelo G. The elastic component of normal and dilated ureters in children: chemical and histochemical characterisation. Br J Urol. 1976 Feb;48(1):13-7

8. Hanna MK, Jeffs RD, Sturgess JM, Barkin M. Ureteral structure and ultrastructure. Part III. The congenitally dilated ureter (megaureter). J Urol. 1977 Jan;117(1):24-7

9. Hanna MK, Jeffs RD, Sturgess JM, Barkin M. Ureteral structure and ultrastructure. Part IV. The dilated ureter, clinicopathological correlation. J Urol. 1977 Jan;117(1):28-32.

10. Gosling JA, Dixon JS. Functional obstruction of the ureter and renal pelvis. A histological and electron microscopic study. Br J Urol. 1978 May;50(3):145-52.

11. Medel R Jr, Quesada EM. Ultrastructural characteristics of collagen tissue in normal and congenitally dilated ureter. Eur Urol. 1985;11(5):324-9

12. Lee BR, Partin AW, Epstein JI, Quinlan DM, Gosling JA, Gearhart JP. A quantitative histological analysis of the dilated ureter of childhood. J Urol. 1992 Nov;148(5):1482-6.

13. Gearhart JP, Lee BR, Partin AW, Epstein JI, Gosling JA. A quantitative histological evaluation of the dilated ureter of childhood. II: Ectopia, posterior urethral valves and the prune belly syndrome. J Urol. 1995 Jan;153(1):172-6.

14. Weibel ER. Morphometry of the human lung: the state of the art after two decades. Bull Eur Physiopathol Respir. 1979 SepOct;15(5):999-1013.

15. Gundersen HJ1, Bendtsen TF, Korbo L, Marcussen N, Møller A, Nielsen K, Nyengaard JR, Pakkenberg B, Sørensen FB, Vesterby A, et al. Some new, simple and efficient stereological methods and their use in pathological research and diagnosis. APMIS. 1988 May;96(5):379-94.

16. Mandarim-de-Lacerda CA. Stereological tools in biomedical research. An Acad Bras Cienc. 2003 Dec;75(4):469-86. Epub 2003 Nov 4. Review. Erratum in: An Acad Bras Cienc. 2007 Mar;79(1):51.

17. Kim DS, Noh JY, Jeong HJ, Kim MJ, Jeon HJ, Han SW. Elastin content of the renal pelvis and ureter determines post-pyeloplasty recovery. J Urol. 2005 Mar;173(3):962-6.

18. Gosling JA, Kung LS, Dixon JS, Horan P, Whitbeck C, Levin RM. Correlation between the structure and function of the rabbit urinary bladder following partial outlet obstruction. J Urol. 2000 Apr;163(4):1349-56.

19. Levin RM, Monson FC, Haugaard N, Buttyan R, Hudson A, Roelofs M, Sartore S, Wein AJ. Genetic and cellular characteristics of bladder outlet obstruction. Urol Clin North Am. 1995 May;22(2):263-83. 
Neves F et al.

\section{Acknowledgements}

This study was supported by grants from the National Council for Scientific and Technological Development (CNPqBrazil) and the Foundation for Research Support of Rio de Janeiro (FAPERJ).

\section{Correspondence:}

Francisco J.B. Sampaio, MD

Urogenital Research Unit

Av. 28 de Setembro, 87 - Fundos - FCM - térreo.

20551-030 Rio de Janeiro, RJ, , Brazil

Fax: + 5521 2587-6121

sampaio.uerj@gmail.com

1Research performed at Urogenital Research Unit, Biomedical Center, State University of Rio de Janeiro, Brazil. 